'Departamento de Imagenología, Facultad de Medicina Clínica Alemana-Universidad del Desarrollo. Santiago, Chile. ${ }^{2}$ Departamento de Imagenología, Hospital Padre Hurtado. Santiago, Chile.

${ }^{3}$ Departamento de Cirugía Vascular, Facultad de Medicina Clínica Alemana-Universidad de

Desarrollo. Santiago, Chile. aResidente de Radiología

Trabajo no recibió financiamiento Los autores declaran no tener conflictos de interés.

Recibido el 5 de noviembre de 2018, aceptado el 3 de mayo de 2019.

Correspondencia a: Giancarlo Schiappacasse Faúndes Vitacura 5951, Vitacura. Santiago, Chile. gschiappacasse@gmail.com

\section{Disección espontánea de arterias viscerales: reporte de una serie de casos}

\author{
GIANCARLO SCHIAPPACASSE ${ }^{1}$, JAVIER CACHO ${ }^{2}$, \\ MARÍA FERNANDA TAPIA ${ }^{1, a}$, FERNANDO IBÁÑEZ ${ }^{3}$
}

\section{Spontaneous dissection of visceral arteries. A series of cases}

Background: Spontaneous dissections of visceral arteries are rare and usually secondary to other disease. There is paucity of information about their natural course. Aim: To describe the imaging, clinical characteristics and follow-up of spontaneous visceral artery dissections diagnosed at our institution. Material and Methods: We report a series of 14 patients in whom a spontaneous dissection of a visceral artery was diagnosed on abdominal angio-CT between 2010 and 2018. Clinical features and evolution were recorded. Results: Isolated lesions of the celiac axis were the most common finding. Multiple territories were involved in $14 \%$ of cases. A dissection flap was observed in four cases, a hematoma-dissection complex in seven and an aneurysmal dilatation associated with the dissection in three. Of the 10 patients who were followed at our institution, $90 \%$ had stabilization or partial regression of the imaging findings. In the remaining case, new dissection events were observed. All cases were managed conservatively, and no death was reported. Conclusions: In this series of patients, spontaneous dissection of visceral arteries had a benign, favorable course, requiring only conservative management.

(Rev Med Chile 2019; 147: 1122-1127)

Key words: Aneurysm, Dissecting; Computed Tomography Angiography; Mesenteric Artery, Superior.

\section{L}

a disección espontánea de las arterias viscerales del abdomen es una entidad rara, siendo más frecuentes las formas secundarias a trauma, vasculitis o bien como extensión de una disección aórtica. Dentro de las causas de disección espontánea de las arterias viscerales se describen patologías poco frecuentes, como la mediolisis arterial segmentaria, la displasia fibromuscular y aun más infrecuente, la necrosis quística de la media $^{1}$. El primer reporte de casos de disección espontánea secundaria a una mediolisis arterial segmentaria fue en 1976 por Slavin et al, en una serie de 3 autopsias en donde la muerte de los pacientes fue ocasionada por la rotura de aneurismas disecantes de arterias musculares viscerales, cuya base anatomopatológica era la presencia de una arteriopatía caracterizada por lisis parcial o completa de la túnica media ${ }^{2}$. Desde entonces se han publicado múltiples artículos sobre esta entidad, sin embargo, la fisiopatología aún no es completamente esclarecida. Dentro de las hipótesis que se manejan es que el evento gatillante de la mediolisis sería un proceso vasoconstrictor del lecho esplácnico ${ }^{3}$.

Menos conocido todavía es el curso evolutivo de estas lesiones en el tiempo. Gran parte de la bibliografía en este aspecto se limita a reportes de casos, con un seguimiento variable de hasta 2 años $^{4}$.

El objetivo de nuestro trabajo es describir las características clínicas e imagenológicas de una serie de 14 casos de disección espontánea de las arterias viscerales y su evolución en el tiempo. 
Seguimiento en disección espontánea de arterias viscerales - G. Schiappacasse et al.

\section{Materiales y Métodos}

Estudio descriptivo retrospectivo, aprobado por el comité de ética de la institución. Se incluyeron todos los casos de disecciones espontáneas de las arterias viscerales pesquisados por angioTC de abdomen (ATA) en nuestra institución durante un periodo de 8 años, desde enero de 2010 a marzo de 2018. Los exámenes fueron realizados en dos tomógrafos computados multicorte (Toshiba Aquilion One [Toshiba Corporation, Tokio, Japón] y SOMATON Definition AS [Siemens Healthineers, Erlangen, Alemania]) según el protocolo de angiografía de nuestra institución. Algunos casos fueron derivados para ATA tras la sospecha en estudios no angiográficos (tomografía computada [TC] de abdomen con contraste en fase venosa y ecografía). Dieciséis casos fueron recolectados en total, 2 se eliminaron porque se demostró factor causal (vasculitis). De los 14 restantes, 10 fueron derivados para ATA luego de la sospecha inicial en TC de abdomen con contraste y ecografía abdominal solicitados por dolor, en 2 casos fue hallazgo de exámenes realizados por otro motivo en pacientes asintomáticos y en solo 2 casos la ATA fue solicitada directamente ante sospecha clínica.

De cada caso se registraron las características demográficas, manifestaciones clínicas y hallazgos imagenológicos al diagnóstico y al seguimiento, los cuales fueron tabulados.

El seguimiento imagenológico fue variable, siendo inexistente en 3 casos y de hasta 7 años en 1 caso.
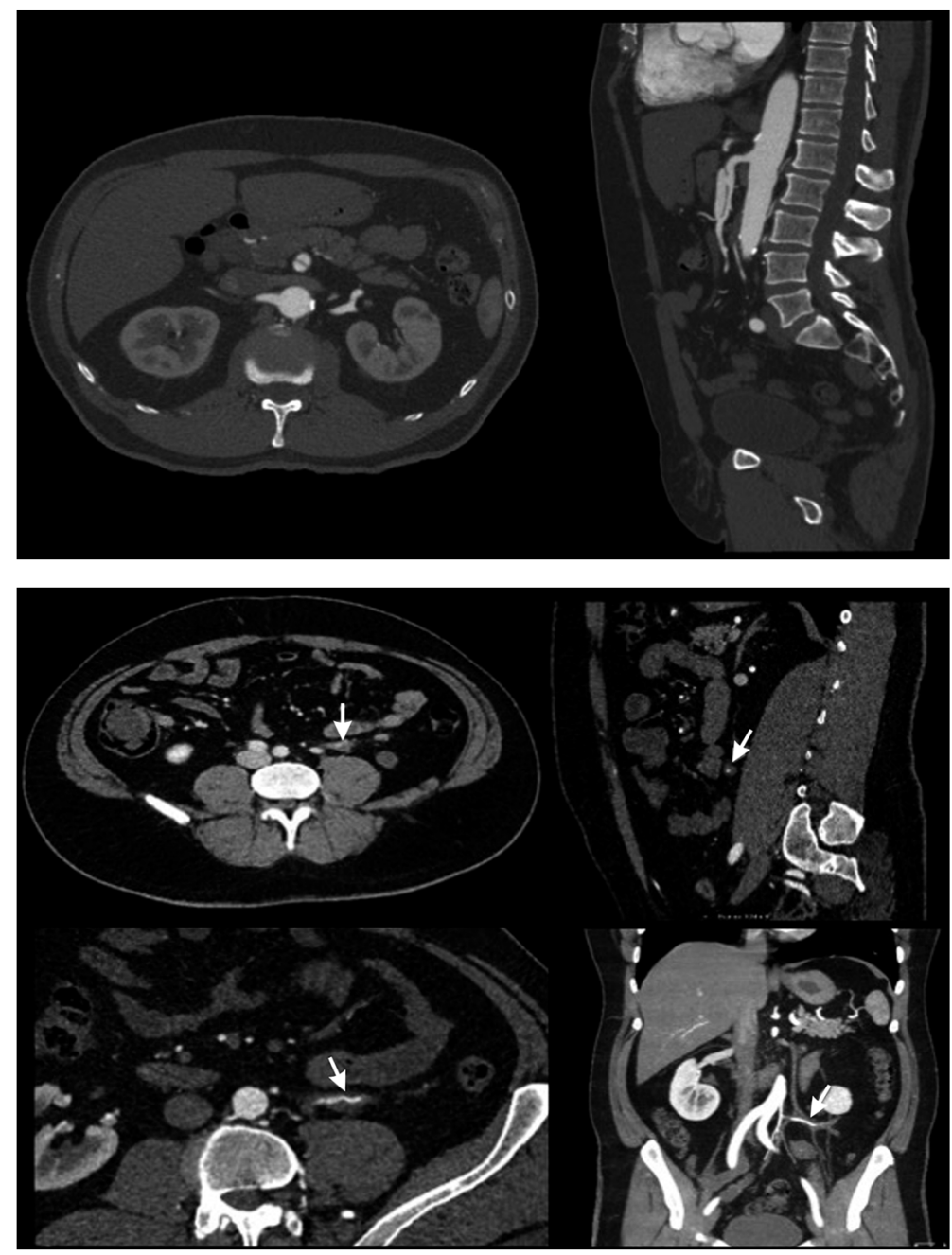

Figura 1. AngiotC de abdomen y pelvis, corte axial y coronal. Se observa un aneurisma-disección de la arteria mesentérica superior, evidenciando un falso lumen y un flap de disección.

Figura 2. AngioTC de abdomen y pelvis, cortes axiales, coronales y sagital. Se evidencia un trombo mural excéntrico de la arteria cólica izquierda, rama de la arteria mesentérica inferior, concordante con un complejo hematoma-disección. 
Seguimiento en disección espontánea de arterias viscerales - G. Schiappacasse et al.

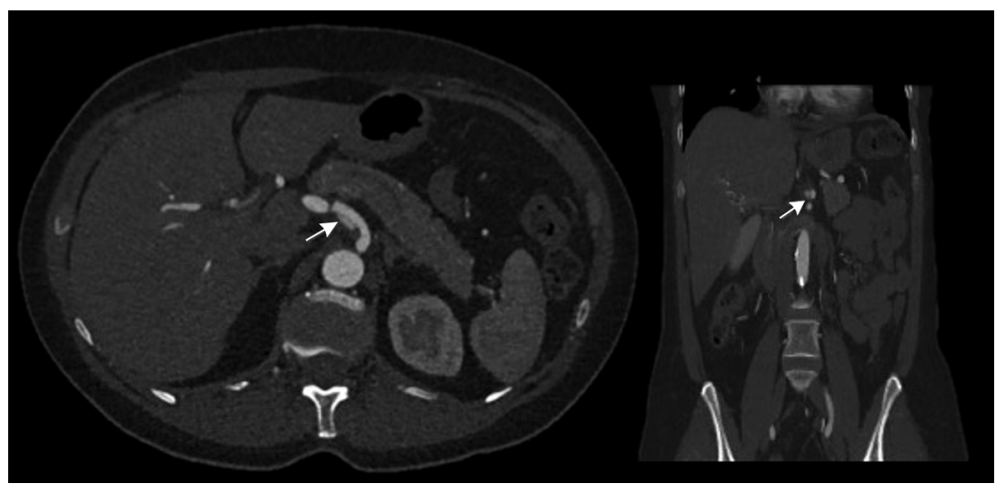

Figura 3. AngioTC de abdomen y pelvis, en corte axial y coronal. Se reconoce una disección del tronco celiaco con un falso lumen (flechas).

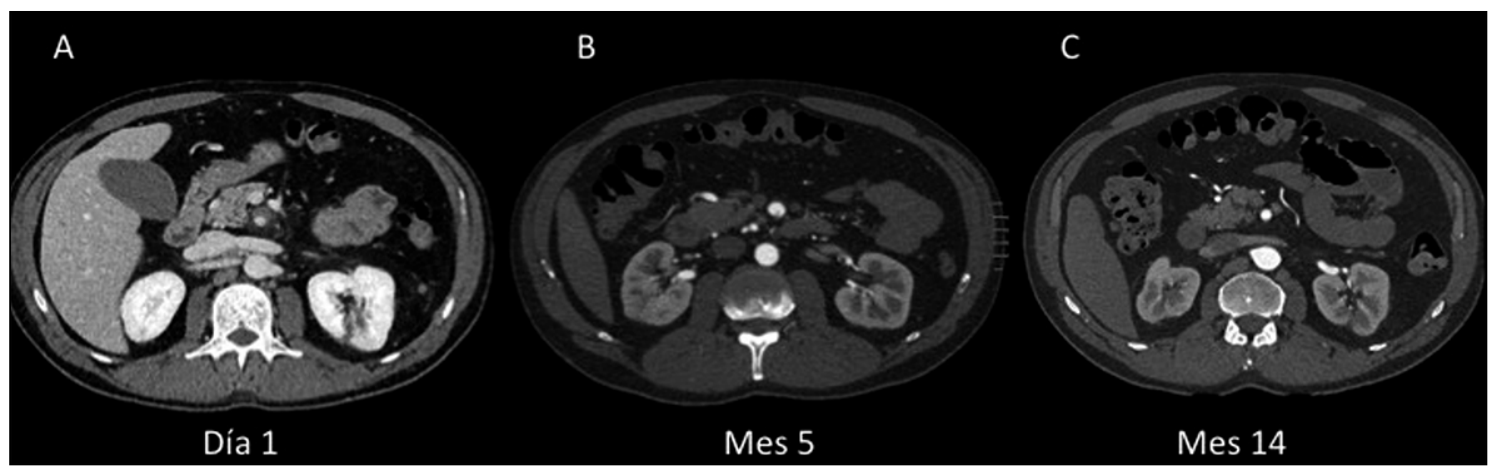

Figura 4. A. TC de abdomen y pelvis con contraste, corte axial, se evidencia disección localizada de la arteria mesentérica superior con trombo mural, que determina una obstrucción mayor a $50 \%$ en su lumen. B. AngioTC de abdomen y pelvis, cortes axiales. Control evolutivo al mes 5, con resolución del trombo mural y visualización del flap de disección. C. AngioTC de abdomen y pelvis, corte axial, donde no se identifica el flap de disección.

\section{Resultados}

Los datos clínicos, hallazgos radiológicos, conducta terapéutica y seguimiento están resumidos en la Tabla 1. La edad media de presentación fue de 55,4 años (rango 37-70 años); 57,1\% de los pacientes era de sexo masculino.

La forma más frecuente de presentación fue dolor abdominal, presente en $78,6 \%$ de los casos, sospechándose origen vascular en solo dos de estos. Los pacientes del grupo restante fueron asintomáticos, pesquisándose incidentalmente en estudios realizados por otro motivo.

En cuanto a los hallazgos imagenológicos, el compromiso de un territorio fue lo más frecuente $(85,7 \%)$, en $50 \%$ de los casos afectó al tronco celíaco, seguido por el compromiso de la arteria mesentérica superior $(41,7 \%)$. En un caso hubo compromiso aislado de la arteria cólica izquierda.

Hubo dos casos en donde existió afectación concomitante de más de un territorio, en el primero del tronco celíaco y arterias renales y en el segundo se agregó además compromiso de la arteria mesentérica superior.

Los hallazgos morfológicos fueron en 4 casos flap de disección aislado, en 7 casos un complejo hematoma-disección y en los 3 restantes, dilatación aneurismática concomitante a la disección. 3 pacientes presentaron pseudoaneurismas asociados.

En cuanto a la terapia, 8 fueron tratados con anticoagulación y 5 con antiagregantes plaquetarios, haciéndose el traslape a anticoagulación 
Seguimiento en disección espontánea de arterias viscerales - G. Schiappacasse et al.

Tabla 1. Resumen de la serie de casos

\begin{tabular}{|c|c|c|c|c|c|c|}
\hline $\begin{array}{l}\text { Pacien- } \\
\text { tes }\end{array}$ & Edad & Sexo & $\begin{array}{l}\text { Hallazgos } \\
\text { clínicos }\end{array}$ & $\begin{array}{c}\text { Hallazgos } \\
\text { CT-vasculares }\end{array}$ & Terapia & Seguimiento \\
\hline 1 & 68 & $\mathrm{~F}$ & $\begin{array}{l}\text { Dolor abdominal } \\
\text { de inicio brusco }\end{array}$ & Disección de la AMS & $\begin{array}{l}\text { Antiagregantes } \\
\text { plaquetarios }\end{array}$ & Sin control \\
\hline 2 & 50 & $\mathrm{~F}$ & $\begin{array}{l}\text { Dolor abdominal } \\
\text { inespecífico }\end{array}$ & $\begin{array}{l}2 \text { territorios. Complejo } \\
\text { hematoma disección } \\
\text { del TC, con formación } \\
\text { de pseudoaneurisma. } \\
\text { Aneurisma sacular de la } \\
\text { arteria renal izquierda }\end{array}$ & $\begin{array}{l}\text { Antiagregantes } \\
\text { plaquetarios }\end{array}$ & $\begin{array}{l}3 \text { años. Trombosis parcial } \\
\text { del pseudoaneurisma, res- } \\
\text { to sin cambios. Aneurisma } \\
\text { renal estable }\end{array}$ \\
\hline 3 & 38 & M & $\begin{array}{l}\text { Dolor abdominal } \\
\text { post-prandial }\end{array}$ & $\begin{array}{l}3 \text { territorios. Complejo } \\
\text { hematoma disección del } \\
\text { TC, de las arterias rena- } \\
\text { les. Complejo hematoma } \\
\text { disección de la AMS en } \\
\text { un segundo tiempo }\end{array}$ & Anticoagulación & $\begin{array}{l}7 \text { años. Dilatación aneu- } \\
\text { rismática del TC en los } \\
\text { primeros } 4 \text { meses, luego } \\
\text { normalización progresiva } \\
\text { del calibre. Ectasia arteria } \\
\text { renal izquierda, persiste } \\
\text { flap de disección. AMS } \\
\text { evoluciona con flap crónico }\end{array}$ \\
\hline 4 & 70 & M & $\begin{array}{l}\text { Dolor epigástrico } \\
\text { crónico }\end{array}$ & $\begin{array}{l}\text { Disección-dilatación } \\
\text { aneurismática de la AMS }\end{array}$ & Anticoagulación & 3 meses. Sin cambios \\
\hline 5 & 60 & $\mathrm{~F}$ & $\begin{array}{l}\text { Dolor abdominal } \\
\text { de inicio brusco }\end{array}$ & $\begin{array}{l}\text { Complejo hematoma } \\
\text { disección del TC }\end{array}$ & Anticoagulación & $\begin{array}{l}1 \text { mes. Resorción hemato- } \\
\text { ma, irregularidad parietal }\end{array}$ \\
\hline 6 & 41 & M & $\begin{array}{l}\text { Dolor abdominal } \\
\text { inespecífico }\end{array}$ & $\begin{array}{l}\text { Complejo hematoma } \\
\text { disección de la AMS }\end{array}$ & Anticoagulación & $\begin{array}{l}3 \text { meses. Persiste flap de } \\
\text { disección. Ectasia AMS }\end{array}$ \\
\hline 7 & 60 & M & Asintomático & $\begin{array}{l}\text { Disección-dilatación } \\
\text { aneurismática del TC }\end{array}$ & Sin tratamiento & 3 años. Estable \\
\hline 8 & 61 & M & $\begin{array}{l}\text { Dolor abdominal } \\
\text { inespecífico }\end{array}$ & Disección TC & Anticoagulación & 6 meses. Estable \\
\hline 9 & 37 & M & $\begin{array}{l}\text { Dolor abdominal } \\
\text { inespecífico }\end{array}$ & $\begin{array}{l}\text { Complejo hematoma } \\
\text { disección AMI }\end{array}$ & Anticoagulación & 1 mes. Sin cambios \\
\hline 10 & 59 & $\mathrm{~F}$ & $\begin{array}{l}\text { Dolor abdominal } \\
\text { postprandial }\end{array}$ & Disección TC & $\begin{array}{l}\text { Antiagregantes } \\
\text { plaquetarios }\end{array}$ & $\begin{array}{l}1 \text { mes. Trombosis lumen } \\
\text { falso }\end{array}$ \\
\hline 11 & 77 & $\mathrm{~F}$ & Sin antecedentes & $\begin{array}{l}\text { Disección-dilatación } \\
\text { aneurismática del TC }\end{array}$ & Sin antecedentes & Sin control \\
\hline 12 & 53 & M & $\begin{array}{l}\text { Dolor abdominal } \\
\text { inespecífico }\end{array}$ & $\begin{array}{l}\text { Complejo hematoma } \\
\text { disección del TC }\end{array}$ & $\begin{array}{l}\text { Anticoagulación } \\
\text { inicial, luego } \\
\text { antiagregación } \\
\text { plaquetaria }\end{array}$ & $\begin{array}{l}2 \text { años. Resorción hema- } \\
\text { toma, persiste flap de } \\
\text { disección }\end{array}$ \\
\hline 13 & 64 & M & Asintomático & Disección de la AMS & $\begin{array}{l}\text { Antiagregantes } \\
\text { plaquetarios }\end{array}$ & Sin control \\
\hline 14 & 37 & $\mathrm{~F}$ & $\begin{array}{l}\text { Dolor abdominal } \\
\text { y hematoquezia }\end{array}$ & $\begin{array}{l}\text { Complejo hematoma } \\
\text { disección de la AMS }\end{array}$ & Anticoagulación & Sin control \\
\hline
\end{tabular}

en uno de ellos. No se dispuso de datos respecto al tratamiento del paciente restante. Ninguno de los pacientes fue sometido a procedimientos invasivos.

Se cuenta con seguimiento imagenológico de
10 pacientes. En $40 \%$ de los casos, los hallazgos imagenológicos regresaron parcialmente y en $40 \%$ se mantuvieron estables. Veinte por ciento restante presentó la siguiente evolución: un caso tuvo un segundo evento de disección de un territorio 
distinto 2 años después, y en el otro, el complejo hematoma-disección evolucionó a dilatación aneurismática de los vasos afectados, los cuales se normalizaron en calibre en los controles posteriores. De los pseudoaneurismas, se trombosaron completamente en un paciente, parcialmente en otro paciente y no se dispone del seguimiento del tercer paciente.

Como complicaciones observadas durante el seguimiento, un paciente desarrolló un infarto esplénico y otro un infarto renal. Ninguno de nuestros pacientes presentó hemorragia intraabdominal ni isquemia intestinal, tampoco hubo muertes producto de la enfermedad, hasta nuestro conocimiento.

\section{Discusión}

Pese a que los primeros casos de disección espontánea de las arterias fueron descritos hace más de 40 años, todavía se conoce poco respecto a esta entidad, particularmente sobre el manejo y evolución de las lesiones en estos pacientes ${ }^{4}$. Corresponde a una entidad rara, probablemente subdiagnosticada por la difícil sospecha clínica. Muchas veces el diagnóstico es evidente en el estudio imagenológico no dirigido en el contexto de dolor abdominal.

Para plantear el diagnóstico de una disección espontánea primero se deben descartar causas secundarias como trauma, vasculitis o infecciones mediante una buena historia clínica y exámenes complementarios ${ }^{5,6}$. El principal diagnóstico diferencial de las disecciones espontáneas de las arterias viscerales es la displasia fibromuscular y mediolisis arterial segmentaria, las cuales se diferencian según el perfil demográfico, la clínica y la distribución de los territorios arteriales afectados ${ }^{3}$. El diagnóstico final solo puede ser alcanzado tras la biopsia.

La displasia fibromuscular frecuentemente afecta mujeres jóvenes, habitualmente asintomáticas. Los territorios más comúnmente afectados son el carotídeo y renal, en forma de irregularidad parietal de aspecto "arrosariado". El compromiso de otras arterias viscerales es más raro y las disecciones son una complicación poco frecuente en este contexto.

La mediolisis arterial segmentaria, en cambio, se ve en pacientes de mayor edad, habitualmente hombres, que debutan con disección de arterias viscerales ${ }^{3}$.

El conocimiento de la evolución de la enfermedad en estos pacientes se limita a reportes de casos aislados y pequeñas series de casos. En nuestro trabajo se cuenta con el seguimiento clínico e imagenológico de 10 pacientes, en 4 de ellos por más de 2 años. El 80\% de los pacientes presentó estabilidad o resolución, ya sea completa o parcial, de los hallazgos imagenológicos. De los 2 casos restantes, uno presentó inicialmente dilatación aneurismática de los vasos afectados, sin embargo, en los controles posteriores el diámetro vascular regresó a la normalidad. Hubo solo un caso que presentó un segundo evento de disección, con compromiso de un territorio vascular diferente a el inicial, sin embargo, las alteraciones nuevamente regresaron parcialmente en los controles posteriores, sin requerir de intervención. En ninguno de los casos de nuestra serie se identificaron complicaciones como rotura y sangrado peritoneal o isquemia intestinal como se describe en la literatura ${ }^{4,7}$ y solo en dos casos se constató isquemia de los territorios afectados (infarto esplénico y renal).

El tratamiento fue conservador en todos los casos, ya sea con antiagregantes plaquetarios o bien anticoagulantes, no requiriéndose de manejo quirúrgico o endovascular como lo reportado en otras series ${ }^{4}$, ya que no existieron complicaciones hemorrágicas agudas. Hasta nuestro conocimiento, no hubo muertes asociadas a la enfermedad, hallazgo que difiere con la literatura, en donde se describe una mortalidad de hasta $50 \%{ }^{8}$.

La principal limitación de nuestro trabajo es que, al igual que el resto de las series ${ }^{9}$, el número de pacientes que se siguieron fue reducido y que no se pudo realizar un seguimiento completo de los pacientes, perdiéndose potencial información del manejo y evolución. Tampoco contamos con la confirmación diagnóstica por medio de biopsia.

\section{Conclusión}

La disección espontánea de arterias viscerales es una entidad poco frecuente, que es subdiagnosticada por un bajo índice de sospecha clínica y que habitualmente presenta una evolución favorable con el tratamiento conservador según lo observado en nuestra serie de casos. 


\section{Referencias}

1. Slavin R. Segmental arterial mediolysis: A clinical-pathologic review, its role in fibromuscular dysplasia and description and differential diagnosis of the masquerader-muscular artery cystic necrosis. WJCD 2013; 3: 64-81.

2. Slavin R, González-Vitale JC. Segmental mediolytic Arteritis - A Clinical Pathologic Study. Lab Invest 1976; 35: 23-9.

3. Alhalabi K, Menias C, Hines R. Mamoun I, Naidu S. Imaging and clinical findings in segmental arterial mediolysis. Abdom Radiol 2017; 42: 602-11.

4. Michael M, Widmer U, Wildermuth S, Barghorn A, Duewell S, Pfammatter T. Segmental Arterial Mediolysis-CTA Findings at Presentation and Follow-Up. AJR Am J Roentgenol 2006; 187: 1463-9.
5. DeCarlo C, Ganguli S, Borges JC, Schainfeld RM, Mintz AJ, Mintz J et al. Presentation, treatment, and outcomes in patients with spontaneous isolated celiac and superior mesenteric artery dossection. Vasc Med 2017; 22 (6): 505-11.

6. Woolard JD, Ammar AD. Spontaneous dissection of the celiac artery: A case report. J Vasc Surg 2007; 45: 1256-8.

7. Slavin R. Segmental Arterial Mediolysis: Course, Sequelae, Prognosis and Pathologic-Radiologic Correlation. Cardiovasc Pathol 2009; 18: 352-60.

8. Chao CP. Segmental Arterial Mediolysis. Semin Intervent Radiol 2009; 26: 224-32.

9. Kalva SP, Somarouthu B, Jaff MR, Wicky S. Segmental Arterial Mediolysis: Clinical and Imaging Features at Presentation and During Follow-up. J Vasc Interv Radiol 2011; 22: 1380-7. 